\title{
Delineating the role of elF2a in retinal degeneration
}

\author{
Christopher R. Starr ${ }^{1}$ and Marina S. Gorbatyuk ${ }^{1}$
}

\begin{abstract}
Activation of the unfolded protein response has been detected in various animal models of retinal degeneration. The PERK branch converges on elF2a to regulate protein synthesis. We previously reported that diseased retinas produce less protein as they degenerate. We also proposed that the majority of this reduction in protein synthesis may not be due to control of elF2a. Nevertheless, multiple research groups have reported that modulating elF2a levels may be a viable strategy in the treatment of neurodegenerative diseases. Here, using two genetic approaches, a systemic Gadd34 knockout and a photoreceptor conditional Perk knockout, to alter p-elF2a levels in rd16 mice, we demonstrate not only that degenerating retinas may not use this mechanism to signal for a decline in protein synthesis rates but also that modulation of $\mathrm{p}$-elF2a levels is insufficient to delay retinal degeneration.
\end{abstract}

\section{Introduction}

Inherited retinal degeneration (IRD) is a class of retinal dystrophies in which there is currently no cure and few treatment options available. Activation of the unfolded protein response (UPR) has been detected in multiple animal models of retinal degeneration ${ }^{1-5}$. The UPR is a series of signaling events following endoplasmic reticulum (ER) stress and is signaled through three ER - resident membrane proteins, protein kinase $\mathrm{R}$ like-endoplasmic reticulum kinase (PERK), inositol requiring enzyme 1 (IRE1) and activating transcription factor 6 (ATF6). PERK signals for a halt in translation following ER stress by phosphorylating eukaryotic initiation factor 2 alpha $(\mathrm{eIF} 2 \alpha)^{6,7}$. This translational attenuation of new protein synthesis aims to reduce the burden on the cell and provides cells with an opportunity to refold incorrectly folded proteins and adapt ${ }^{7}$.

Multiple groups have reported elevated activation of the PERK branch in animal models of retinal degeneration. There are no known roles of eIF2 $\alpha$ other than protein synthesis regulation, so it has been assumed that its

Correspondence: Marina S. Gorbatyuk (mgortk@uab.edu)

'Department of Optometry and Vision Science, The University of Alabama at Birmingham, School of Optometry, Birmingham, AL, USA

Edited by N. Bazan phosphorylation leads to a substantial attenuation in protein synthesis rates, but no study has functionally and genetically modulated p-eIF $2 \alpha$ in degenerating retinas and subsequently assessed retinal protein synthesis rates and photoreceptor survival. In addition, as we have proposed previously ${ }^{1}$, eIF $2 \alpha$ may not be the primary point of translational control in degenerating retinas. Those findings make us question what role, if any, eIF2 $\alpha$ phosphorylation plays in retinal degeneration.

The phosphorylation of eIF $2 \alpha$ by one or more kinases, together with the alternative translational and transcriptional program has been coined the integrated stress response, or ISR ${ }^{8}$. When eIF2 $\alpha$ is phosphorylated, eIF2 can no longer participate in translation and protein synthesis rates typically go down; however, translation of certain mRNAs with upstream open reading frames (uORFs) is actually promoted in these conditions ${ }^{6}$. One protein known to be selectively translated following eIF $2 \alpha$ phosphorylation is ATF4, a transcription factor that promotes expression of several stress related genes including growth arrest and DNA-damage 34 (GADD34) $^{9}$. GADD34, also known as protein phosphatase 1 regulatory subunit 15A (PPP1R15A), is the stress induced-phosphatase regulatory subunit that recruits protein phosphatase 1 (PP1) and initiates the dephosphorylation of phosphorylated-eIF2 $\alpha(\mathrm{p}-\mathrm{eIF} 2 \alpha)^{9-11}$. 
Therefore, GADD34 is a central component of the feedback loop aiming to restore translation following a transient stress. Another important player of PP1 is CreP (PP1R15B), which is responsible for maintaining basal levels of $\mathrm{p}$-eIF2 $\mathrm{a}^{12}$. Aside from regulating eIF $2 \alpha$ phosphorylation, GADD34 has a multitude of other roles including promoting apoptosis ${ }^{13-16}$. Interestingly, a recent study suggested that PP1/GADD34 may act on a plethora of previously unknown targets ${ }^{13}$.

We previously reported that a majority of eIF $2 \alpha$ phosphorylation is likely due to PKR-like endoplasmic reticulum kinase (PERK) in $r d 16$ mice $^{1}$. Although we also revealed that eIF $2 \alpha$ may not be the primary means by which degenerating retinas control translation ${ }^{1}$, it is still unknown whether this mechanism plays a role in RD. Recently, multiple research groups have demonstrated that diminishing PERK under chronic ER stress can result in improvement of neuronal function and survival in neurodegenerative diseases ${ }^{17-20}$. While important, these studies have not focused on validation of this therapeutic strategy to restore general protein synthesis, first and have not compared a degree of the restoration in the same animal model modulating other regulatory nodes of translation, such as 4E-BP1/2. Therefore, the mentioned studies demonstrate the gap in knowledge in this field and indicates that the role of p-eIF2a under chronic ER stress needs to be examined carefully. In this study, we aim to delineate whether eIF2 $\alpha$ plays a significant role in maintaining protein synthesis under chronic ER stress and therefore, determine if it contributes to progressive retinopathy.

\section{Results}

\section{Strategy to modulate the elF2a activity}

The ability of eIF $2 \alpha$ to regulate protein synthesis and therefore allow the cell to cope with stress depends on its phosphorylation status. Thus, when phosphorylated, eIF2 $\alpha$ mediates the binding of the initiator tRNA-Met to the ribosome in a GTP-dependent manner. In order to modulate its activity, we decided to up- and downregulate its phosphorylation state, which requires either deactivating protein phosphatase (PP1) or the active eIF2 $\alpha$ kinase, PERK. To that end, we generated $r d 16$ Gadd $34^{-1-}$ and $r d 16$ Perk $^{f / f}$ iCre75 mice to access protein synthesis and investigate their contribution to translational modulation during chronic activation of the ISR.

\section{Increasing the p-elF2a does not further diminish protein synthesis but delays retinal degeneration via GADD34 ablation}

We previously found an activated ISR in the retinas of rd16 mice at P15 and P20 as shown by elevations in markers including p-eIF2 $\alpha$, ATF4, and C/EBP homologous protein (CHOP). $r d 16$ is a mouse model with a rapidly degenerating retina due a spontaneous deletion in the Cep290 gene, the most frequently mutated gene in Leber congenital amaurosis. Interestingly, GADD34 was only upregulated at P20. Activation of the ISR coincided with a decline in translation rates in $r d 16$ mice at $\mathrm{P} 15^{1}$. To assess whether the phosphorylation status of eIF $2 \alpha$ plays a role in retinal degeneration, we generated $\mathrm{rd} 16 \mathrm{Gadd} 34^{-/-}$ mice. As expected, when compared to retinas of $r d 16$ mice,

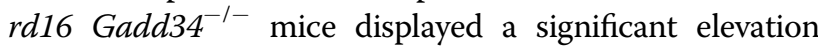
(62\%) in phosphorylated eIF2 $\alpha$ levels (Fig. 1a, b). We next sought whether the elevation in p-eIF $2 \alpha$ levels was associated with a change in levels of nascent protein synthesis. Protein synthesis rates were detected using the Surface Sensing of Translation (SUnSET) method ${ }^{21}$. Our adaptation of the SUnSET method to detect nascent peptide synthesis in the retina was described previously ${ }^{1}$. To our surprise, the drastic elevation in p-eIF2 $\alpha$ levels did not correspond to a significant change in protein synthesis rates (Fig. 1c, d).

To determine whether knocking out Gadd34 had any impact on retinal degeneration, we first counted photoreceptor nuclei in the outer nuclear layer $(\mathrm{ONL})$. To our surprise, we found that $\mathrm{rd16}$ Gadd34 ${ }^{-1-}$ mice had significantly more nuclei in their ONL at P18 than $r d 16$ mice (Fig. 2a, b). However, there was no significant difference in electroretinography (ERG) amplitudes between the two groups (Fig. 2c, d), which could be due to non-uniform protection of degenerating photoreceptors across the retina, which is lost when ERG responses of the entire retina are averaged as done in our experiment. The rapid rate of retinal degeneration exhibited by these mice ${ }^{1,22-26}$, and the fact that they do not develop normal outer segments $^{22,27}$, makes them particularly challenging to rescue. There was also not a significant difference in ERG amplitudes between C57BL/6J and Gadd34 ${ }^{-1-}$ mice at P25 (Fig. S1). We then assessed Müller cell gliosis in these groups by staining retinal cryosections with antibody against glial fibrillary acidic protein (GFAP) and vimentin. GFAP and vimentin are intermediate filament forming proteins. GFAP is highly expressed in gliotic Müller cells and vimentin is expressed in all Müller glia, giving us a way to differentiate between Müller cells and astrocytes ${ }^{28}$. We found that while $r d 16$ mice had apparent radial GFAP branching corresponding to Müller cells, GFAP staining was largely limited to the inner limiting membrane, and therefore astrocytes, in $\mathrm{rd} 16 \mathrm{Gadd34^{-1- }}$ and C57BL/6 mice (Fig. 3a). This suggests that at P18, rd16 Gadd34 $4^{-1-}$ mice demonstrate less Müller cell gliosis than $r d 16$ mice. We then evaluated cell death in the ONL in these groups by TUNEL analysis. At P15, we found that the retinas of rd16 mice had significantly less apoptotic cell death than the retinas of $\mathrm{rd} 16 \mathrm{Gadd}_{34}{ }^{-1-}$ mice (Fig. 3b, c). At P18 however, rd16 Gadd34 ${ }^{-1-}$ had significantly more cell death than $r d 16$, indicating that apoptosis is delayed in the ONL of $r d 16$ Gadd34 $4^{-1-}$ mice. 


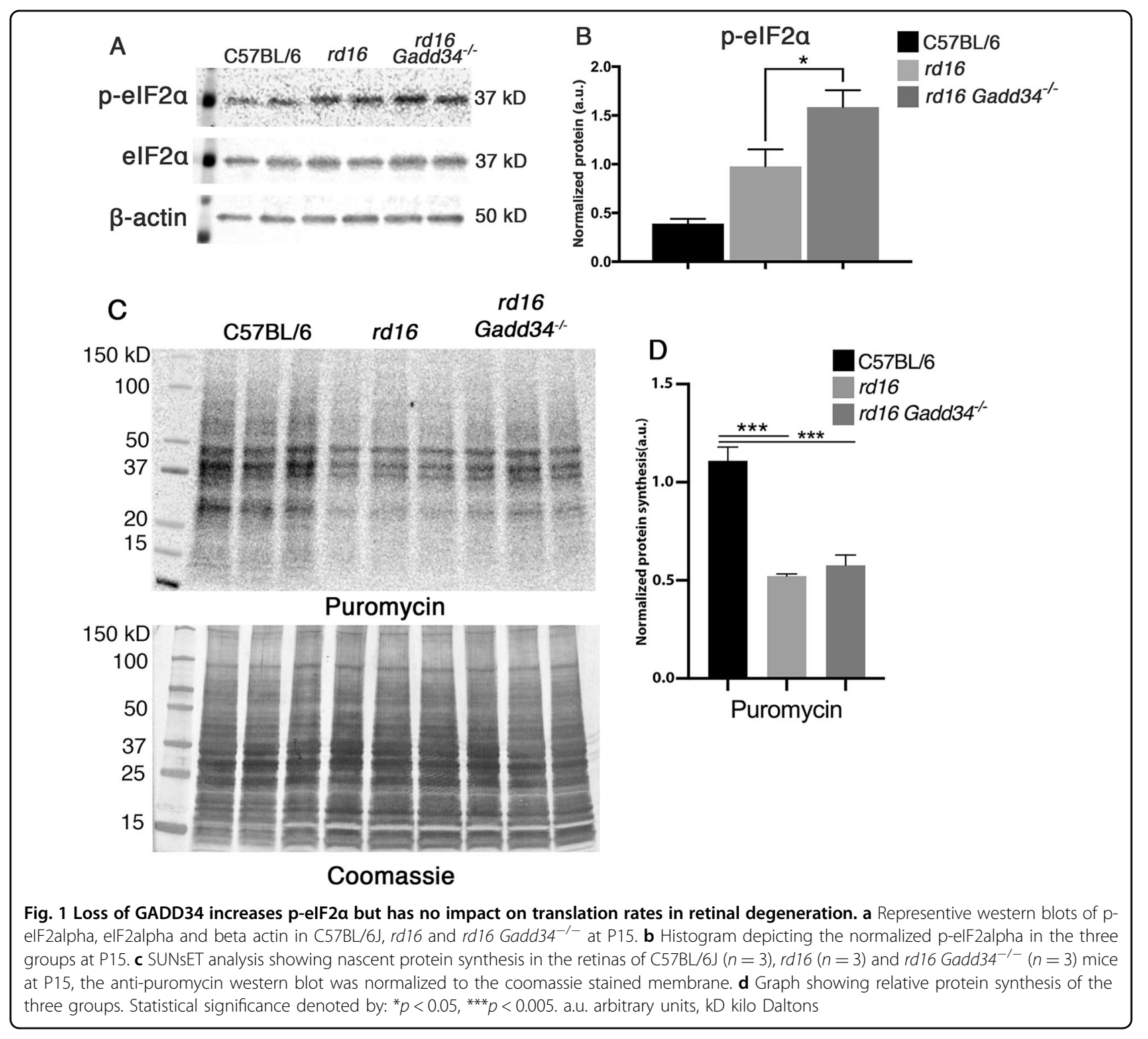

\section{Lowering p-elF2a levels does not restore protein synthesis} and does not delay retinal degeneration

Since higher p-eIF $2 \alpha$ levels in mice lacking Gadd34 were associated with a delay in retinal degeneration in $r d 16$ mice, we next hypothesized that lowering p-eIF2 $\alpha$ levels by targeting PERK would worsen retinal degeneration. We previously proposed that the majority of protein synthesis attenuation seen in the retina during RD may not be due to eIF $2 \alpha$ regulation $^{1}$. In that study, we pharmacologically inhibited PERK in $r d 16$ mice and discovered that while p-eIF $2 \alpha$ levels decreased by about $50 \%$ in treated mice, protein synthesis only recovered by $\sim 17 \%$. Since PERK inhibiting compounds are non-specific ${ }^{29}$ and highly toxic ${ }^{30}$, we decided to implement a genetic model to study the role of PERK and eIF $2 \alpha$ in RD. To that end, we generated Perk $^{f / f}$ iCre75 and $r d 16$ Perk $^{f / f}$ iCre75 mice.
iCre75 mice house a Cre transgene under the control of a mouse opsin promoter, which gives rise to rod photoreceptor specific knockout of floxed genes ${ }^{31}$, therefore enabling us to study the role of the PERK in photoreceptors. We first assessed PERK levels in the retinas of rd16 and $r d 16$ Perk $^{f / f}$ iCre75 mice by western blot analysis and IHC. Proteins were extracted from whole retina lysates and subjected to western blot analysis. As expected, PERK levels in total retina protein lysates were significantly lower $(\sim 34 \%)$ in $r d 16$ Perk $^{f / f}$ iCre75 than $r d 16$ mice (Fig. 4a, b). In addition, compared to $r d 16$, there was a prominent reduction in PERK immunofluorescence in both the cell bodies surrounding the photoreceptor cell nuclei in the ONL and inner segments, of rd16 Perk $^{f / f}$ iCre75 mice (Fig. 4c), whereas the immunofluorescence of the INL and GCL is more consistent between the two 


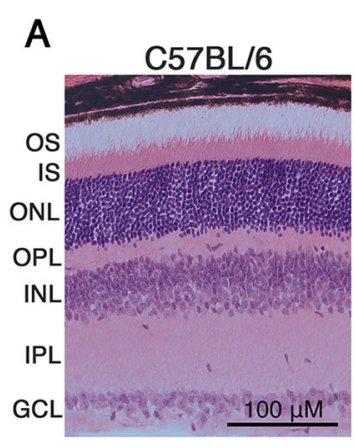

C

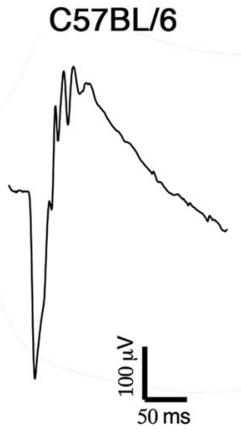

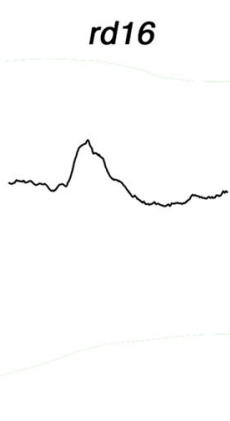
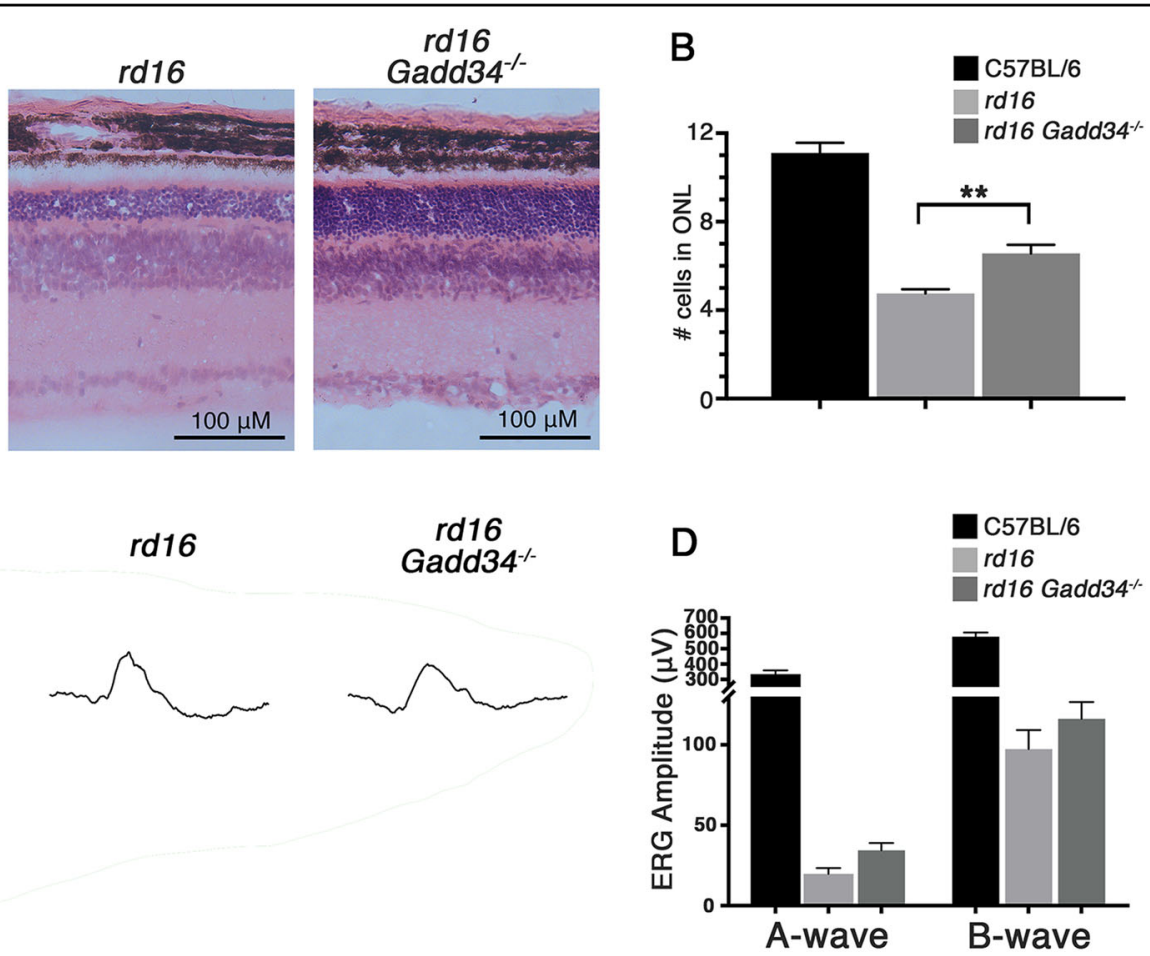

Fig. 2 Knocking out Gadd34 delays retinal degeneration. a Representative images of H\&E stained sections at P18. b Graph depicting mean number of nuclei in the ONL of C57BL/6J $(n=3), r d 16(n=7)$ and $r d 16$ Gadd34-/- $(n=10)$ as counted by a masked investigator. ONL from $400 \mu M-$ wide micrographs were counted. c Averaged ERG waveforms of dark-adapted mice following a $10 \mathrm{~dB}$ flash. $\mathbf{d}$ Histogram showing average a- and bwave ERG amplitudes of C57BL/6J $(n=4), r d 16(n=6)$ and $r d 16$ Gadd34 ${ }^{-/-}(n=10)$ mice at P17. Statistical significance denoted by: ${ }^{* *} p<0.01$. OS outer segments, IS inner segments, ONL outer nuclear layer, OPL outer plexiform layer, INL inner nuclear layer, IPL inner plexiform layer, GCL ganglion cell layer

groups. Importantly, the immunofluorescent regions in the micrographs are consistent with ER localization ${ }^{32}$. We next assessed whether this decrease in PERK levels corresponded to a reduction in levels of p-eIF2 $\alpha$ in $r d 16$ Perk $^{f / f}$ iCre75 mice. To that end, p-eIF2 $\alpha$ levels were assessed in retinal lysates isolated from C57BL/6J, $r d 16$ and $r d 16$ Perk $^{f / f}$ iCre75 mice at P15. As expected, the retinas of $r d 16$ mice exhibited a significant increase in $\mathrm{p}$ eIF2 $\alpha$ levels compared to C57BL/6J (Fig. 5a, b). However, rd16 Perk $^{f / f}$ iCre75 retinas had significantly lower p-eIF $2 \alpha$ levels $(\sim 32 \%)$ than the $r d 16$ mice, demonstrating that although levels were still significantly higher $(\sim 17 \%)$ in the retinas of $r d 16$ Perk $^{f / f}$ iCre75 mice than C57BL/6J mice, loss of Perk was associated with an almost complete normalization in eIF2 $\alpha$ phosphorylation (Fig. 5b). We next assessed whether the decrease in p-eIF $2 \alpha$ levels was associated with a change in protein synthesis rates. Interestingly, the lower p-eIF $2 \alpha$ levels in $r d 16$ Perk $^{f / f}$ iCre75 mice were not associated with a significant increase in protein synthesis rates (Fig. 5c, d). Together with the translation data from $r d 16$ Gadd $34^{-1-}$ mice and our previous report showing that PERK inhibition only marginally restores protein synthesis rates in the retina ${ }^{1}$, these results demonstrate that eIF $2 \alpha$ regulation is not the primary means by which degenerating retinas control protein synthesis rates. Nevertheless, a group previously reported that the phosphorylation state of eIF2 $\alpha$ may play a role in retinal degeneration ${ }^{5}$. Therefore, we next compared the retinal viability of these three groups. We assessed the retinal function of these groups using scotopic ERG at P17. We did not detect a significant difference in a- or b-wave amplitude between $r d 16$ and $r d 16$ Perk $^{f / f}$ iCre75 (Fig. 6a, b). Importantly, ERG amplitudes of C57BL/6J mice and Perk ${ }^{f / f}$ iCre75 mice were not significantly different at this time point. Further, there was also no detectable difference in ERG amplitudes between C57BL/6J mice and Perk ${ }^{f / f}$ iCre75 mice at P60 (Fig. S2). We then assessed apoptotic photoreceptor cell death in the ONL at P15 by TUNEL analysis. To our surprise, we found that there was not a significant difference in the number of TUNEL-positive nuclei between between $r d 16$ and $r d 16$ Perk $^{f / f}$ iCre75 (Fig. 6c, d). 


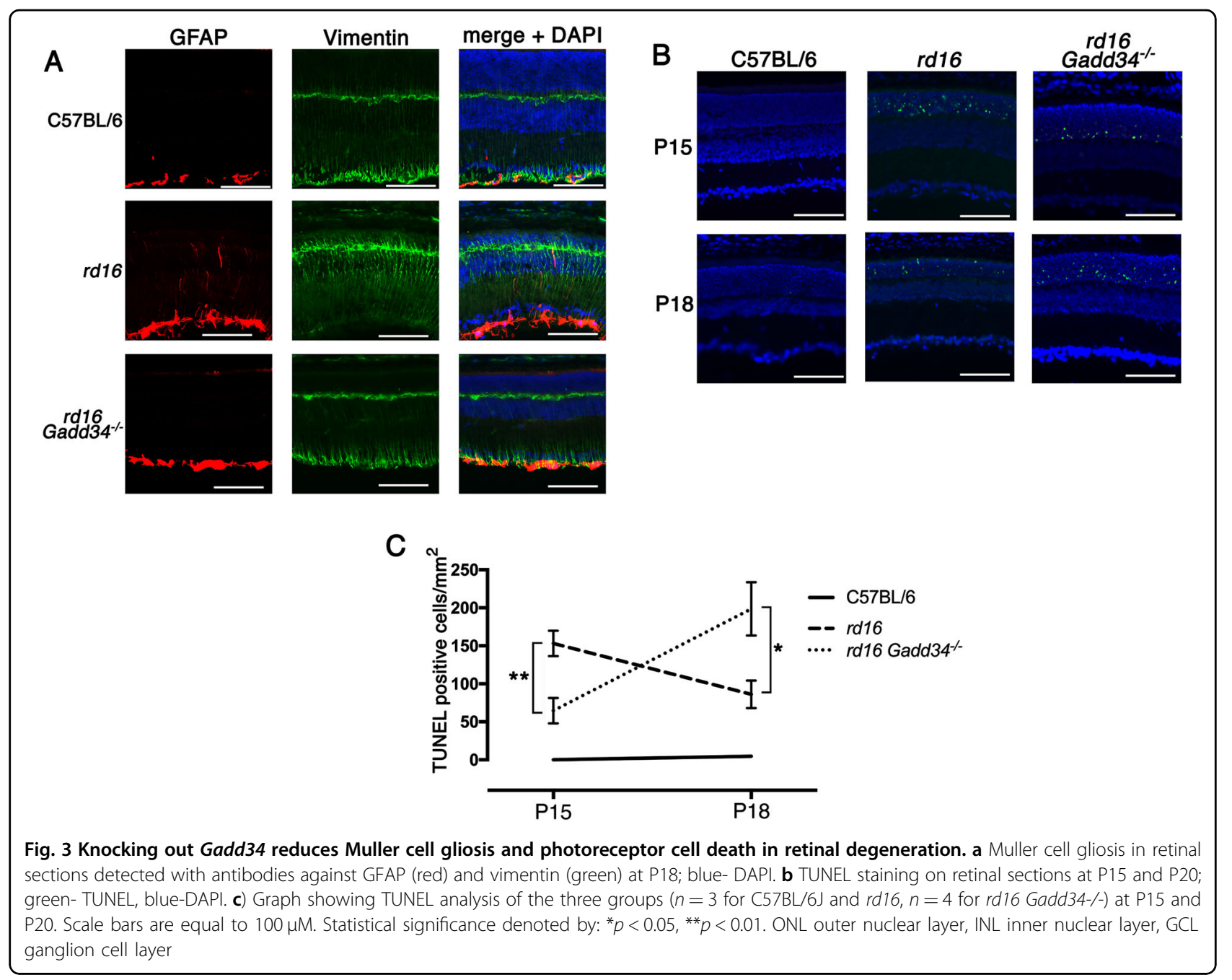

\section{Loss of Gadd34 is associated with AKT activation in degenerating retinas}

Since knocking out Gadd34 resulted in a significant increase in p-eIF $2 \alpha$ levels in $r d 16$ mice that correlated with a delay in retinal degeneration, we expected the loss of PERK in photoreceptors to have the opposite effect due to lower p-eIF2 $\alpha$ levels. This made us wonder if something else could be occurring in the retinas of $r d 16$ Gadd $34^{-l-}$ mice. Although eIF2 $\alpha$ does not have any known function outside of protein synthesis regulation, GADD34 has a multitude of proposed functions outside of eIF2 $\alpha$ regulation ${ }^{13-15,33}$. One particular function of GADD34 is its well-documented role as a pro-apoptotic protein $^{14,16,34}$. One pro-apoptotic duty of GADD34 is its inhibition of AKT activation ${ }^{14,16,34}$. We previously reported that $r d 16$ mice have diminished levels of $\mathrm{p}$-AKT at $\mathrm{P} 15^{1}$. To determine whether the delay of RD in $r d 16$ Gadd $34^{-1-}$ mice could have be due to enhanced AKT activation, we assessed levels of p-AKT (Ser473) in the retinas of $\mathrm{C} 57 \mathrm{BL} / 6, r d 16$ and $r d 16$ Gadd34 $4^{-1-}$ mice at
P15. Interestingly, compared to $r d 16$ mice, the retinas rd16 Gadd34-/- mice had significantly elevated levels of p-AKT (Fig. 7a, b), which may indicate that the knockout of Gadd34 improves photoreceptor viability by stimulating AKT signaling rather than through regulation of eIF $2 \alpha$.

\section{Discussion}

Multiple reports have proposed regulating the ISR to ameliorate retinal degeneration ${ }^{5,35-37}$. In the present study, we provide evidence suggesting that regulation of eIF2 $\alpha$ alone may not be sufficient to significantly change protein synthesis rates or impact retinal cell viability. In fact, reducing p-eIF $2 \alpha$ levels by knocking out Perk in retinal photoreceptors had no impact on photoreceptor survival in $r d 16$ mice. However, an elevation in p-eIF2 $\alpha$ levels in the retinas of $r d 16$ Gadd34 $4^{-1-}$ mice was associated with a delay in photoreceptor cell death even in the absence of a change in the levels of protein synthesis. There are no known functions of eIF $2 \alpha$ outside of protein 


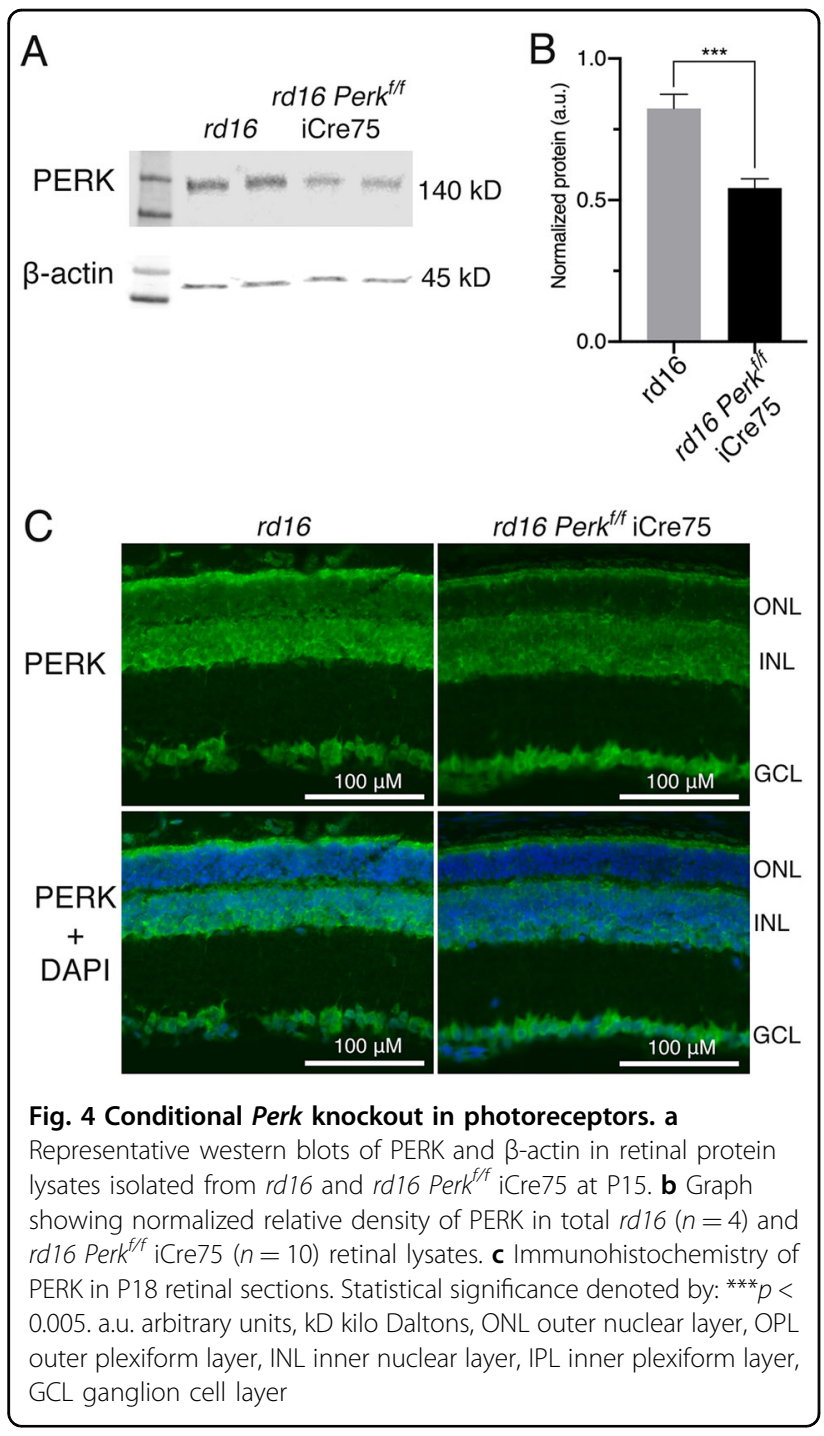

synthesis regulation, but there are many other functions of GADD34 ${ }^{13-16,33}$. Therefore, it is likely that the delay in RD observed $r d 16$ Gadd34 $4^{-1-}$ is due to the loss of GADD34 rather than the change in p-eIF2 $\alpha$ levels. In fact, there have been a number of studies that have implicated GADD34 in the induction of apoptosis ${ }^{14,16,34}$. It is possible that GADD34 ablation could selectively regulate translation of anti-apoptotic proteins in degenerating retinas, which could be due to a $5^{\prime}$-cap independent, or internal ribosome entry site (IRES) mechanism. One wellcharacterized pro-apoptotic role of GADD34 is its ability to inhibit membrane-translocation of AKT and its subsequent activation ${ }^{14}$. AKT has a multitude of pro-survival functions as it directly phosphorylates a number of proapoptotic and apoptotic proteins. We showed that the retinas of rd16 mice lacking Gadd34 experienced a delay in photoreceptor cell death that coincides with an elevation in AKT phosphorylation, which is consistent with the reported role of Gadd34 in AKT inactivation.

The viability of Gadd34-1- mice has been reported previously $^{38-40}$, so there was little surprise that mice lacking GADD34 exhibited normal ERG amplitudes. However, we found it particularly surprising that PERK does not seem to be needed for photoreceptors. Whereas Gadd $34^{-1-}$ was knocked out systemically in our study, we used a Cre-recombinase driven by a mouse opsin promoter to knock out Perk in photoreceptors. Using a conditional knockout of Perk allowed us to study the role of PERK in photoreceptors without the issue of pancreatic atrophy, however, the iCre75 transgenic mouse does not show recombination around loxP sites until P7 and recombination persists until as late as $\mathrm{P} 18^{31}$. Therefore, whether PERK is important for photoreceptor development could not be assessed in the present study.

The results of this study suggest that regulating eIF $2 \alpha$ phosphorylation may not be enough to delay apoptosis. However, knocking out GADD34 resulted in a delay in retinal degeneration but had no impact on retinal function as assessed by ERG. This could be due to the $r d 16$ mouse model having a photoreceptor ciliopathy ${ }^{22,23}$, as these animals do not form normal outer segments ${ }^{22,27}$. This makes $r d 16$ mice particularly challenging to rescue. It would perhaps be better to assess the role of GADD34 in another animal model such as one that does not degenerate so rapidly or more directly assess the role of GADD34 in $r d 16$ retinas under simultaneous inhibition of p-eIF2 $\alpha$ by ISRIB compound. The role of PERK under chronic ISR activation should also be verified by using other animal models, even though this kinase appears to be redundant in wild type retinas. Another possible reason that p-eIF $2 \alpha$ modulation may not affect protein synthesis is the eIF2 guanine exchange factor, eIF2B. eIF2 is the translation initiation factor that brings the initiator methionine to the pre-initiation complex. eIF2B is the rate-limiting step regulating formation of the preinitiation complex. It is possible that in $r d 16$ retinas, eIF2B could have saturated affinity of binding to p-eIF2 $\alpha$, thus, limiting the therapeutic targeting of p-eIF $2 \alpha$. In addition, if eIF2 is truly only regulating a small portion of protein synthesis in degenerating retinas, some other translation factor-initiator tRNA complex must have high activity in the retina.

Recently, research conducted in animal models of PD, $\mathrm{AD}$ and tauopathies have demonstrated the therapeutic effect from targeting the PERK arm of the UPR including eIF2 $\alpha$, GADD34 and PERK ${ }^{18,41-44}$. Through modulation of these factors, these studies have shown improvement of neuronal function and viability. The results of the current study however, aimed at validating the PERK UPR arm as a therapeutic target and suggested that this signaling may not be the most efficient strategy to protect neuronal cell 


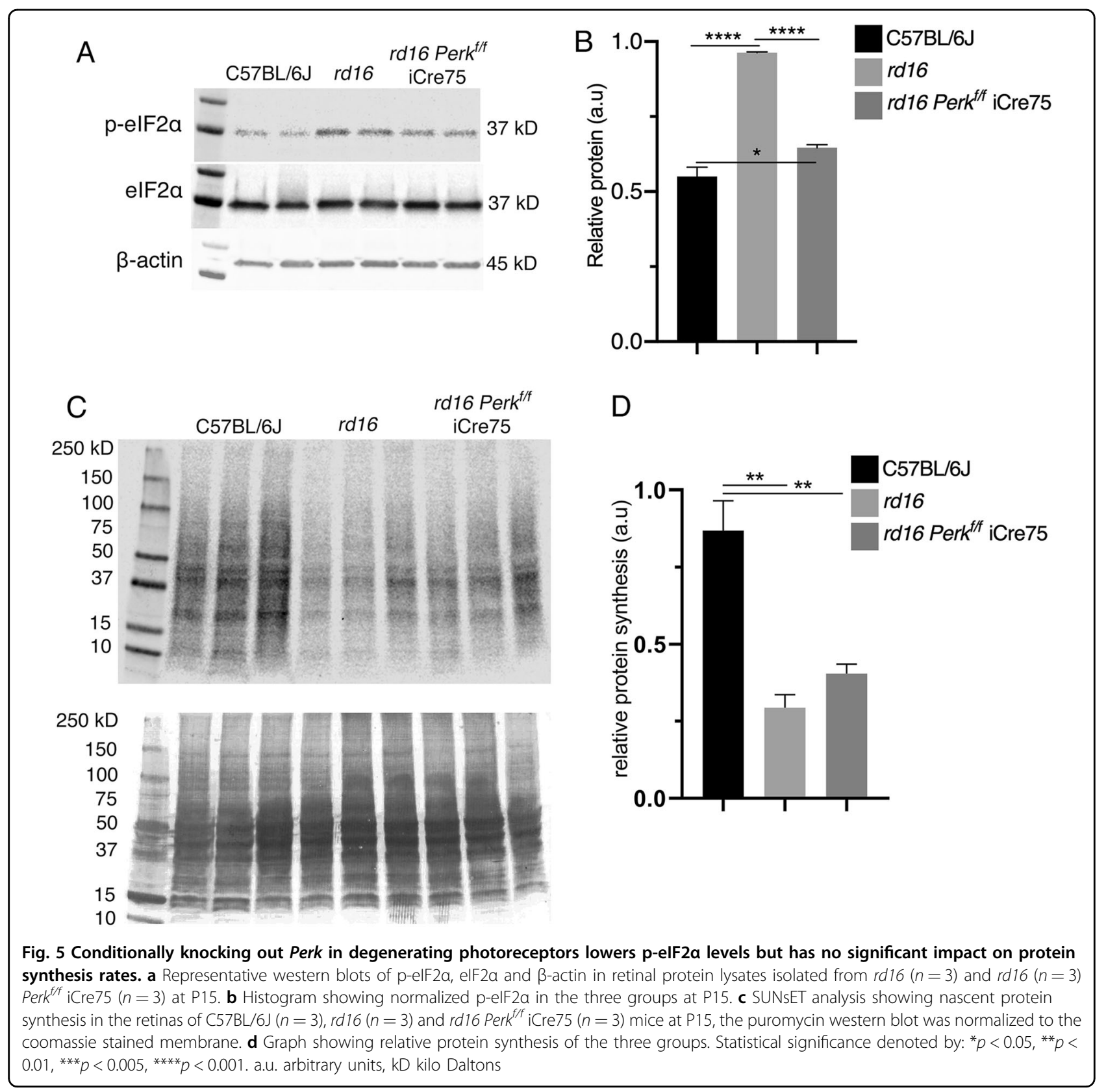

death. Previously, we have reported diminished levels of $\mathrm{AKT} \rightarrow \mathrm{mTOR} \rightarrow 4 \mathrm{E}-\mathrm{BP} 1$ signaling in $r d 16$ retinas ${ }^{1}$. Therefore, investigation of this signaling will significantly enhance our molecular understanding of translational control in degenerating retinas.

\section{Materials and methods}

Animals

All animal experiments followed a protocol (IACUC\#131109793) approved by the University of Alabama at Birmingham Institutional Animal Care and Use Committee (IACUC), and conformed to guidelines set by the Association of Research in Vision Science and Ophthalmology. BXD24/TyJ-Cep290 ${ }^{r d 16} / \mathrm{J}$ (000031, rd16), Eif2ak3 ${ }^{\mathrm{tm} 1.2 \mathrm{Drc}} / \mathrm{J}$ (023066, Perk ${ }^{f / f}$ )and C57BL/6J (000664) mice were obtained from Jackson Laboratory (Bar Harbor, ME). Gadd34 KO mice were provided by Dr. David Ron and have been previously described ${ }^{45}$. iCre75 mice have been described ${ }^{31}$. These mice were crossed with $r d 16$ mice to yield the genotypes mentioned in this article. Genotyping of each of these mice has been mentioned previously. Mice were housed in a facility with a 12-hour light/dark cycle. Mice had free access to a standard diet and water. At the time points specified in the following 

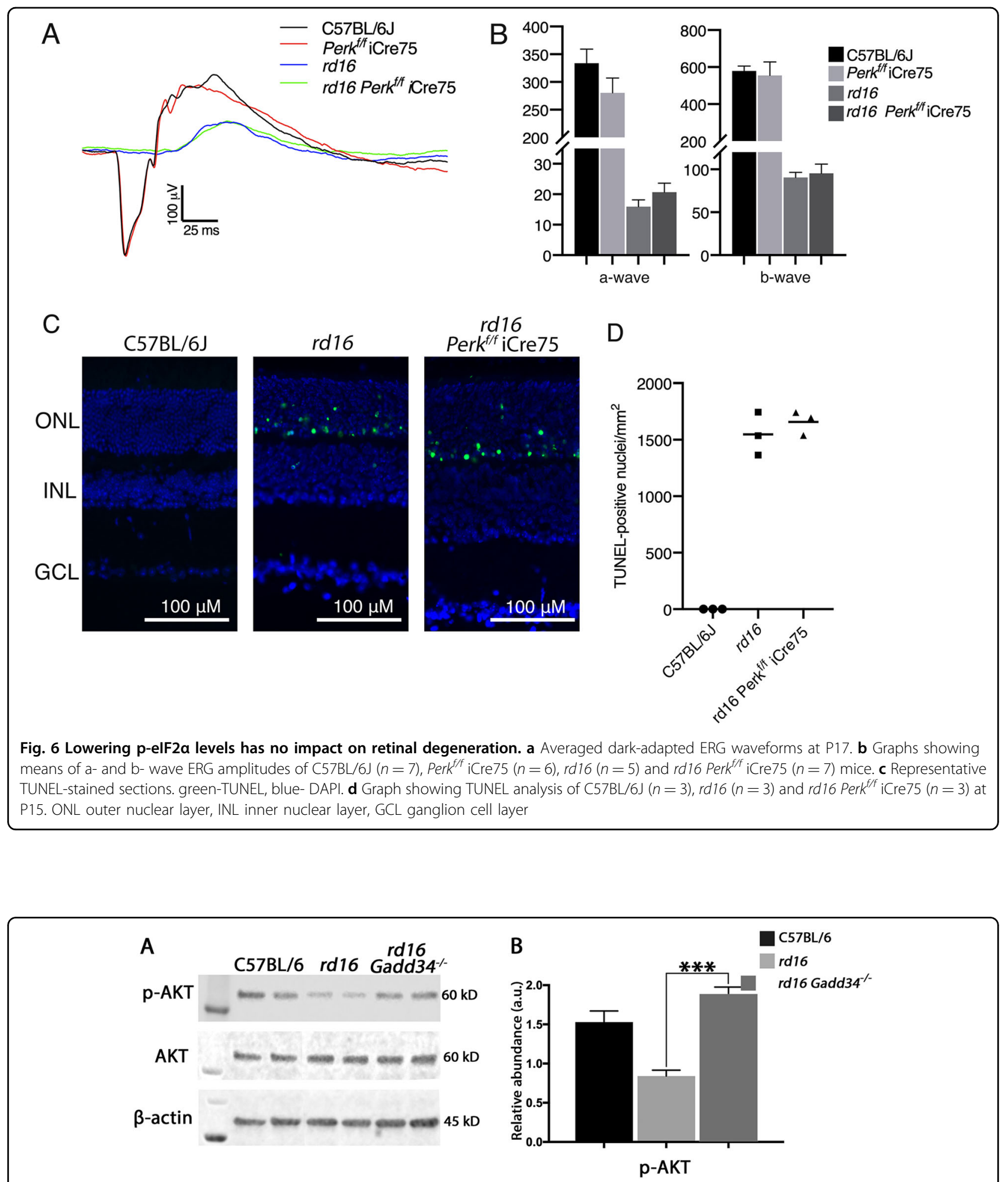

Fig. 7 Knocking out Gadd34 is associated with an elevation in AKT activation in degenerating retinas. a Western blots of p-AKT, AKT and $\beta$-actin. b Analysis of normalized AKT phosphorylation in the retinal lysates isolated from C57BL/6J $(n=5), r d 16(n=4)$ and $r d 16$ Gadd34 ${ }^{-1-}(n=5)$ mice at P15. Statistical significance denoted by: ${ }^{* *} p<0.005$. a.u. arbitrary units, kD kilo Daltons 
sections and text body, mice were euthanized by $\mathrm{CO}_{2}$ asphyxiation followed by cervical dislocation.

\section{Western Blotting}

For western blot analyses, mouse retinas were dissected and homogenized in lysis buffer $(150 \mathrm{mM} \mathrm{NaCl}, 1.0 \%$ Triton X-100, $0.5 \%$ sodium deoxycholate, $0.1 \%$ SDS, and $50 \mathrm{mM}$ Tris $\mathrm{pH} 8.0$ ). Protein concentrations were estimated by the Bio-Rad protein assay (5000001, Hercules, CA, USA). Protein lysate $(40-60 \mu \mathrm{g})$ were separated by SDS-PAGE and transferred to a PVDF membrane for immunoblotting. Primary antibodies specific for phosphoeIF2 $\alpha$ (p-S51, 3398, rabbit, lot: 6), eIF2 $\alpha$ (9722, rabbit, lot: 15), p-AKT (p-S473, 4060, rabbit, lot: 14), AKT (4691, rabbit, lot: 20) were purchased from Cell Signaling Technologies (Danvers, MA, USA). The anti- $\beta$-actin primary antibody (A2228, mouse) was purchased from Sigma Aldrich (St louis, MO, USA). Secondary antibodies (WesternSure HRP goat anti-mouse IgG: 926-80010, WesternSure HRP goat anti-rabbit IgG: 926-80011, and IRDye $800 \mathrm{CW}$ Goat anti-mouse: 925-32210) were purchased from LI-COR (Lincoln, NE, USA). Probed membranes were imaged on a LICOR Fc imaging system. Relative band densities were measured using ImageJ software. Westerns were normalized to $\beta$-actin. Phosphorylated proteins were normalized to their nonphosphorylated counterparts.

\section{Histology and immunohistochemistry}

Eyes were enucleated at P15 or P18 and washed in phosphate buffered saline. Eyes were then placed in $4 \%$ paraformaldehyde (diluted in PBS from 16\% paraformaldehyde, electron microscopy sciences 15710; Hatfield, PA) for $30 \mathrm{~min}$ at room temperature. After $30 \mathrm{~min}$, a needle (33G) was inserted at the limbus to create a small hole for $4 \%$ paraformaldehyde to enter. These eyes were then fixed in $4 \%$ paraformaldehyde at $4{ }^{\circ} \mathrm{C}$ for $4 \mathrm{~h}$ or overnight, depending on the assay for which they were used (4 h: IHC, H\&E. overnight: TUNEL). Fixed eyes were washed with PBS and immersed in 30\% sucrose for at least $1 \mathrm{~h}$. Eyes were then removed from sucrose and embedded in Tissue-Tek O.C.T. compound (VWR: 25608-930) and kept at $-80^{\circ} \mathrm{C}$ for $1 \mathrm{~h}$. Cryomolds were then equilibrated to the temperature inside $\left(-21^{\circ} \mathrm{C}\right)$ the cryostat sectioning system (Leica CM 1510S; Leica, Buffalo Grove, IL, USA). $12 \mu \mathrm{M}$ eye sections were cut using a cryostat tissue sectioning system. Sections were stained with hematoxylin and eosin (Electron Microscopy Sciences: 26754-1A, 26762-01). A masked investigator counted the number of rows of nuclei in the ONL in $400 \mu \mathrm{M}$-wide micrographs. Anti-GFAP (G3893) was purchased from Sigma-Aldrich and anti-vimentin (5741) was purchased from Cell Signaling Technologies. The antibody against PERK (sc-377400 AF488) was purchased from Santa Cruz Biotechnology (Dallas, TX, USA). Terminal deoxynucleotidyl transferase (TdT) dUTP NickEnd Labeling (TUNEL)-staining (Click-it plus TUNEL assay, ThermoFisher scientific, C10617) was performed on retinal sections following instructions from the manufacture. Sections were counterstained with DAPI (Vector Laboratories, H-1200). TUNEL-positive nuclei were counted automatically using the RETINA analysis toolkit for ImageJ. Images of sections were acquired using a Zeiss Axio-plan2 fluorescent microscope.

\section{Analysis of nascent protein synthesis}

The SUnSET method has been described previously ${ }^{1,21}$. For in vivo analysis of nascent peptide synthesis, mice were intraperitoneally injected with puromycin (puromycin dihydrochloride; Santa Cruz Biotechnology, CAS 58-58-2) at a dosage of $0.04 \mu \mathrm{mol} / \mathrm{g}$ body mass. Retinas were harvested $30 \mathrm{~min}$ after the puromycin injection. Proteins were then extracted in RIPA buffer and protein concentrations were estimated as stated above in the western blotting section. Between 40 and $60 \mu \mathrm{g}$ of protein were separated by SDS-PAGE and then transferred to a PVDF membrane. Membranes were then incubated with an antibody specific to Puromycin (MABE343, mouse, lot: 2861354), which was purchased from EMD Millipore. A secondary antibody specific to IgG2a (goat anti-mouse peroxidase affinipure IgG, Fc $\gamma$ Subclass 2a Specific: 115-035-206, Jackson Immuno Research Laboratories Inc; West Grove, PA, USA) was used to prevent detection of endogenous immunoglobulin heavy and light chains. As a loading control, membranes were stained with coomassie blue R-250 (Bio-Rad: \#1610436). After antipuromycin immunoblotting, membranes were washed with distilled water and then incubated in coomassie staining solution for $60 \mathrm{~s}$. Membranes were then destained (ethanol/acetic/water; 5:1:4 proportion) for 10-30 min and air dried before scanning on a Kyocera Taskalfa copier. Relative densities of entire lanes were measured using ImageJ software. Puromycin densities were normalized to their corresponding coomassie densities.

\section{Electroretinography}

Mice were dark adapted overnight. Electroretinography was conducted using a LKC BIGSHOT ERG instrument. Briefly, mice anesthetized with ketamine and xylazine and then their eyes were dilated. Once dilated, the animals were placed on the instrument. Mice were then exposed to 5 flashes of $25 \mathrm{~cd} . \mathrm{s} / \mathrm{m}^{2}$ in $45 \mathrm{~s}$ intervals. ERG waveforms were then analyzed using LKC EM software.

\section{Statistics}

Student $t$ test was used to compare two groups and ANOVA was carried on comparisons made between three 
or more groups. All statistics were performed using Graphpad Prism 8 software.

\section{Acknowledgements}

This work was supported by the National Institutes of Health Grant R01 EY027763. We sincerely thank Dr. David Ron for generously providing Gadd34-/- mice.

\section{Conflict of interest}

The authors declare that they have no conflict of interest.

\section{Publisher's note}

Springer Nature remains neutral with regard to jurisdictional claims in published maps and institutional affiliations.

Supplementary Information accompanies this paper at (https://doi.org/ 10.1038/s41419-019-1641-y).

Received: 18 March 2019 Revised: 12 April 2019 Accepted: 8 May 2019 Published online: 28 May 2019

\section{References}

1. Starr, C. R., Pitale, P. M. \& Gorbatyuk, M. Translational attenuation and retinal degeneration in mice with an active integrated stress response. Cell Death Dis 9, 484 (2018)

2. Lebon, C., Behar-Cohen, F. \& Torriglia, A. Cell death mechanisms in a mouse model of retinal degeneration in Spinocerebellar Ataxia 7. Neuroscience $\mathbf{4 0 0}$ 72-84 (2019).

3. Wang, J., Saul, A., Cui, X., Roon, P. \& Smith, S. B. Absence of sigma 1 receptor accelerates photoreceptor cell death in a murine model of retinitis pigmentosa. Invest. Ophthalmol. Vis. Sci. 58, 4545-4558 (2017).

4. Lenin, R. et al. Critical role of endoplasmic reticulum stress in chronic endothelial activation-induced visual deficits in tie2-tumor necrosis factor mice. J. Cell. Biochem. 119, 8460-8471 (2018).

5. Athanasiou, D. et al. The role of the ER stress response protein PERK in rhodopsin retinitis pigmentosa. Hum. Mol. Genet. https://doi.org/10.1093/hmg/ ddx370. (2017).

6. Gorbatyuk, M. \& Gorbatyuk, O. Review: retinal degeneration: focus on the unfolded protein response. Mol Vision 19, 1985-1998 (2013).

7. Hetz, C. \& Saxena, S. ER stress and the unfolded protein response in neurodegeneration. Nat Rev. Neurol. 13, 477-491 (2017).

8. Taniuchi, S., Miyake, M., Tsugawa, K., Oyadomari, M. \& Oyadomari, S. Integrated stress response of vertebrates is regulated by four elF2alpha kinases. Sci. Rep. $\mathbf{6}$, 32886 (2016).

9. Ma, Y. \& Hendershot, L. M. Delineation of a negative feedback regulatory loop that controls protein translation during endoplasmic reticulum stress. J. Biol. Chem. 278, 34864-34873 (2003).

10. Kojima, E. et al. The function of GADD34 is a recovery from a shutoff of protein synthesis induced by ER stress: elucidation by GADD34-deficient mice. FASEB J. 17, 1573-1575 (2003).

11. Novoa, I., Zeng, H., Harding, H. P. \& Ron, D. Feedback inhibition of the unfolded protein response by GADD34-mediated dephosphorylation of elF2alpha. J. Cell Biol. 153, 1011-1022 (2001).

12. Rojas, M., Vasconcelos, G. \& Dever, T. E. An elF2alpha-binding motif in protein phosphatase 1 subunit GADD34 and its viral orthologs is required to promote dephosphorylation of elF2alpha. Proc. Natl Acad. Sci. USA 112, E3466-E3475 (2015).

13. Dedigama-Arachchige, P. M., Acharige, N. P. N. \& Pflum, M. K. H. Identification of PP1-Gadd34 substrates involved in the unfolded protein response using KBIPS, a method for phosphatase substrate identification. Mol. Omics 14, 121-133 (2018).

14. Farook, J. M. et al. GADD34 induces cell death through inactivation of Akt following traumatic brain injury. Cell Death Dis. 4, e754 (2013).

15. Gu, M. et al. Phosphatase holoenzyme PP1/GADD34 negatively regulates TLR response by inhibiting TAK1 serine 412 phosphorylation. J. Immunol. 192, (2846-2856 (2014).
16. Kang, H. J. et al. Guanabenz Acetate Induces Endoplasmic Reticulum StressRelated Cell Death in Hepatocellular Carcinoma Cells. J. Patholog. Transl. Med. https://doi.org/10.4132/jptm.2019.01.14. (2019).

17. Abisambra, J. F. et al. Tau accumulation activates the unfolded protein response by impairing endoplasmic reticulum-associated degradation. J Neurosci. 33, 9498-9507 (2013).

18. Ma, T. et al. Suppression of elF2alpha kinases alleviates Alzheimer's diseaserelated plasticity and memory deficits. Nat. Neurosci. 16, 1299-1305 (2013).

19. Radford, H., Moreno, J. A., Verity, N., Halliday, M. \& Mallucci, G. R. PERK inhibition prevents tau-mediated neurodegeneration in a mouse model of frontotemporal dementia. Acta Neuropathol. 130, 633-642 (2015).

20. Sharma, $V$. et al. Local Inhibition of PERK enhances memory and reverses agerelated deterioration of cognitive and neuronal properties. J Neurosci. $\mathbf{3 8}$ 648-658 (2018)

21. Goodman, C. A. \& Hornberger, T. A. Measuring protein synthesis with SUnSET: a valid alternative to traditional techniques? Exerc. Sport sciences reviews 41, 107-115 (2013).

22. Chang, B. et al. In-frame deletion in a novel centrosomal/ciliary protein CEP290/NPHP6 perturbs its interaction with RPGR and results in early-onset retinal degeneration in the rd16 mouse. Hum. Mol. Genet. 15, 1847-1857 (2006).

23. Mookherjee, S. et al. A CEP290 C-terminal domain complements the mutant CEP290 of Rd16 mice in trans and rescues retinal degeneration. Cell Rep. 25 611-623.e616 (2018).

24. Murga-Zamalloa, C. A. et al. Accumulation of the Raf-1 kinase inhibitory protein (Rkip) is associated with Cep290-mediated photoreceptor degeneration in ciliopathies. J. Biol. Chem. 286, 28276-28286 (2011).

25. Subramanian, B., Anand, M., Khan, N. W. \& Khanna, H. Loss of Raf-1 kinase inhibitory protein delays early-onset severe retinal ciliopathy in Cep290rd16 mouse. Invest. Ophthalmol. Vis. Sci. 55, 5788-5794 (2014).

26. Zhang, W., Li, L., Su, Q., Gao, G. \& Khanna, H. Gene therapy using a miniCEP290 fragment delays photoreceptor degeneration in a mouse model of leber congenital amaurosis. Hum. Gene Ther. 29, 42-50 (2018).

27. Rachel, R. A. et al. CEP290 alleles in mice disrupt tissue-specific cilia biogenesis and recapitulate features of syndromic ciliopathies. Hum. Mol. Genet. 24 3775-3791 (2015)

28. Lewis, G. P. \& Fisher, S. K. Up-regulation of glial fibrillary acidic protein in response to retinal injury: its potential role in glial remodeling and a comparison to vimentin expression. Int. Rev. Cytol. 230, 263-290 (2003).

29. Rojas-Rivera, D. et al. When PERK inhibitors turn out to be new potent RIPK1 inhibitors: critical issues on the specificity and use of GSK2606414 and GSK2656157. Cell Death Differ. 24, 1100-1110 (2017).

30. Moreno, J. A. et al. Oral treatment targeting the unfolded protein response prevents neurodegeneration and clinical disease in prion-infected mice. Sci. Transl. Med. 5, 206 ra138 (2013).

31. Li, S. et al. Rhodopsin-iCre transgenic mouse line for Cre-mediated rod-specific gene targeting. Genesis 41, 73-80 (2005).

32. Johnson, J. E. Jr. et al. Spatiotemporal regulation of ATP and Ca2 + dynamics in vertebrate rod and cone ribbon synapses. Mol. Vision 13, 887-919 (2007).

33. Song, P. et al. The regulatory protein GADD34 inhibits TRAll-induced apoptosis via TRAF6/ERK-dependent stabilization of myeloid cell leukemia 1 in liver cancer cells. J. Biol. Chem. https://doi.org/10.1074/jbc.RA118.006029. (2019).

34. Lee, J. E., Morrison, W. \& Hollien, J. Hairy and enhancer of split 1 (HES1) protects cells from endoplasmic reticulum stress-induced apoptosis through repression of GADD34. J. Biol. Chem. 293, 5947-5955, (2018).

35. Ghaderi, S. et al. AAV delivery of GRP78/BiP promotes adaptation of human RPE cell to ER stress. J Cell. Biochem. 119, 1355-1367 (2018).

36. Ooe, E. et al. The involvement of ATF4 and S-opsin in retinal photoreceptor cell damage induced by blue LED light. Mol. Vision 23, 52-59 (2017).

37. Bhootada, Y. et al. Limited ATF4 expression in degenerating retinas with ongoing er stress promotes photoreceptor survival in a mouse model of autosomal dominant retinitis pigmentosa. PLOS ONE 11, e0154779 (2016).

38. Nishio, N., Hasegawa, T., Tatsuno, I., Isaka, M. \& Isobe, K. I. Female GADD34 mice develop age-related inflammation and hepatocellular carcinoma. Geriatr. Gerontol. Int. 17, 2593-2601 (2017).

39. Nishio, N. \& Isobe, K. GADD34-deficient mice develop obesity, nonalcoholic fatty liver disease, hepatic carcinoma and insulin resistance. Sci. Rep. 5, 13519 (2015).

40. Patterson, A. D., Hollander, M. C., Miller, G. F. \& Fornace, A. J. Jr. Gadd34 requirement for normal hemoglobin synthesis. Mol. Cell. Biol. 26, 1644-1653 (2006). 
41. Bruch, J. et al. PERK activation mitigates tau pathology in vitro and in vivo. EMBO Mol. Med. 9, 371-384 (2017).

42. Mercado, G. et al. Targeting PERK signaling with the small molecule GSK2606414 prevents neurodegeneration in a model of Parkinson's disease Neurobiol. Dis. 112, 136-148 (2018).

43. Yang, W. et al. Repression of the elF2alpha kinase PERK alleviates mGluR-LTD impairments in a mouse model of Alzheimer's disease. Neurobiol. Aging 41, 19-24 (2016)
44. Halliday, M. et al. Partial restoration of protein synthesis rates by the small molecule ISRIB prevents neurodegeneration without pancreatic toxicity. Cell Death Dis. 6, e1672 (2015).

45. Harding, H. P. et al. Ppp1r15 gene knockout reveals an essential role for translation initiation factor 2 alpha (elF2alpha) dephosphorylation in mammalian development. Proc. Natl Acad. Sci. USA 106, 1832-1837 (2009). 